\title{
Rheumatoid Arthritis-associated Polymorphisms at 6q23 Are Associated with Radiological Damage in Autoantibody-positive RA
}

\author{
JAMES R. MAXWELL, IOANNA MARINOU, KAR-PING KUET, GISELA OROZCO, DAVID J. MOORE, \\ ANNE BARTON, JANE WORTHINGTON, and A. GERRY WILSON
}

\begin{abstract}
Objective. Recent studies have identified 6q23 as an important susceptibility locus for rheumatoid arthritis (RA), with risk alleles at 3 single-nucleotide polymorphisms combining to give an effect size greater than that of these markers individually. We investigated whether these polymorphisms are also associated with disease severity measured by radiological damage.

Methods. We studied 927 patients from a cross-sectional RA cohort. Median Larsen scores (LS) read from radiographs taken at study entry were compared by genotype at rs6920220, rs13207033, and rs5029937 according to a dominant model using negative binomial regression with stratification for autoantibody status.

Results. Median LS was associated with genotype at rs6920220 [LS 31 GG vs 36 GA/AA (p = 0.02) in cyclic citrullinated peptide+ (CCP) RA] and rs13020220 [LS 37 GG vs 29 GA/AA (p = 0.02) in CCP+ RA] only in autoantibody-positive RA, with no association at rs5029937. Association was stronger for these markers in combination [LS 28 vs 42 for lowest vs highest risk genotype combination in rheumatoid factor positivity $(\mathrm{p}=0.007)$, LS 28 vs 37 for anti-CCP+ $(\mathrm{p}=0.01)]$.

Conclusion. Established RA risk markers at 6q23 are associated also with radiographic severity in autoantibody-positive RA; as for susceptibility, the association for these markers in combination is stronger than that for markers alone. (First Release July 15 2012; J Rheumatol 2012;39:1781-5; doi:10.3899/jrheum.120144)
\end{abstract}

Key Indexing Terms:

SINGLE-NUCLEOTIDE POLYMORPHISM RADIOGRAPHY

$6 q 23$ RHEUMATOID ARTHRITIS
DISEASE SEVERITY
Recently reported genetic association studies have identified the $6 q 23$ region as one of the most important susceptibility loci for rheumatoid arthritis (RA). This locus was identified by a genome-wide association study (GWAS) performed by The Wellcome Trust Case Control Consortium ${ }^{1}$. A subsequent British case-control study confirmed the association with RA of rs6920220 located in an intergenic region between the oligodendrocyte lineage transcription factor 3 (OLIG3) and tumor necrosis factor- $\alpha$ induced protein 3

From the Department of Infection and Immunity, Medical School, University of Sheffield, Sheffield; and the Arthritis Research UK Epidemiology Unit, Manchester Academy of Health Sciences, University of Manchester, Manchester, UK.

Funded by the Arthritis Research Campaign.

J.R. Maxwell, MD; I. Marinou, PhD; K-P. Kuet, MD, Department of Infection and Immunity, Medical School, University of Sheffield; G. Orozco, PhD, Arthritis Research UK Epidemiology Unit, Manchester Academy of Health Sciences, University of Manchester; D.J. Moore, Department of Infection and Immunity, Medical School, University of Sheffield; A.C. Barton, MD, PhD; J. Worthington, PhD, Arthritis Research UK Epidemiology Unit, Manchester Academy of Health Sciences, University of Manchester; A.G. Wilson, MD, PhD, Department of Infection and Immunity, Medical School, University of Sheffield.

Address correspondence to Dr. J. Maxwell, Department of Infection and Immunity, Medical School, University of Sheffield, Sheffield S10 2RX, UK. E-mail: J.Maxwell@sheffield.ac.uk

Accepted for publication May 18, 2012.
(TNFAIP3) genes at $6 \mathrm{q} 23.3^{2}$. The association of the region encompassing OLIG3-TNFAIP3 was further replicated in a US study that combined both a case-control study and GWAS in identifying a further independently associated single-nucleotide polymorphism (SNP; rs10499194), providing confirmation that the region is a true RA susceptibility locus $^{3}$. A subsequent fine-mapping study, including the TNFAIP3 gene in addition to the TNFAIP3-OLIG3 intergenic region, has shown that although 3 polymorphisms, rs6920220, rs13207033 (a perfect proxy for rs10499194), and rs5029937, have independent associations with RA, the combination of the risk alleles at rs6920220 and rs5029937 and the absence of the protective allele at rs13207033 has an effect size that is larger than that for any of these SNP individually ${ }^{4}$. This hypothesis was further confirmed in a Spanish cohort in which a combination of markers from the intergenic region and the TNFAIP3 gene was more strongly associated with RA than markers from either locus alone ${ }^{5}$.

In addition to RA susceptibility there is increasing evidence of a genetic contribution to disease severity. A study compared the variance in radiological hand damage in monozygotic and dizygotic twins and pairs of unrelated patients with RA. Assuming a linear relationship between radiological progression and disease duration, variation in

Personal non-commercial use only. The Journal of Rheumatology Copyright @ 2012. All rights reserved. 
joint destruction was highest in unrelated pairs, followed by dizygotic twins, and was smallest between monozygotic twins, supporting a genetic input ${ }^{6}$. HLA-DRB1 alleles are also associated with more severe joint damage in $\mathrm{RA}^{7}$, although recent studies have suggested that this association actually maps to the carriage of anticyclic citrullinated peptide (anti-CCP) antibodies ${ }^{8}$. Genetic associations between interleukin 6 (IL-6) and IL-10 genotypes with radiological damage in rheumatoid factor (RF)- and anti-CCP-positive and negative patients, respectively, have been reported recently by our unit ${ }^{9}$, and carriage of the IL-1B $+3954 \mathrm{~T}$ allele has been associated with more severe structural damage in 2 studies, independent of autoantibody status ${ }^{10,11}$. Association of the 6q23 region with joint damage in RA has recently been reported in a Dutch cohort, in which 2 SNP were associated with rate of joint damage in a cohort of anti-CCP-positive RA patients ${ }^{12}$, but there was no statistical association between rs6920220 and radiological outcome in this study, or with the presence of erosions in an RA inception cohort ${ }^{13}$. A recent study in mice with collagen-induced arthritis also showed significant reduction in tissue destruction in mice treated with A20 (the product of TNFAIP3), delivered via an adenovirus ${ }^{14}$.

We investigated whether the 3 variants confirmed in the 6q23 fine-mapping study to be associated with RA susceptibility are associated with radiological severity alone or in combination and after stratification by autoantibody status.

\section{MATERIALS AND METHODS}

Patients. White patients fulfilling the 1987 American College of Rheumatology (ACR) criteria for the diagnosis of RA and with a minimum disease duration of 3 years $(n=927)$ were recruited from the Royal Hallamshire Hospital, Sheffield, UK, as described ${ }^{15}$. Research ethics committee approval was obtained for the study and all participants gave informed consent.

Radiographs of hands and feet were obtained at study entry, and modified Larsen scores were available for all study subjects. Radiographs of hands and feet were scored blind at study entry by a single musculoskeletal radiologist (DJM) using the modified Larsen score, which provides an integrated measure of bone and cartilage damage in RA. To check whether scoring was consistent, $10 \%$ of radiographs were selected at random and returned for repeat blinded analysis. A weighted kappa score was calculated to quantify the intraobserver variation in the modified Larsen scoring. The weighted kappa score was 0.83 , indicating very good agreement between the initial and repeat scores.

Measurement of antibodies and genotyping. Anti-CCP antibody levels were measured using the Diastat ${ }^{\mathrm{TM}}$ anti-CCP ELISA (Axis-Shield, Cambridgeshire, UK). The semiquantitative protocol was used as recommended by the manufacturer and a cutoff of 5.5 units $/ \mathrm{ml}$ was established based on mean plus 3 standard deviations of values obtained from 100 age-matched control individuals. RF was measured using a nephelometric method on the Dade Behring BN2 nephelometer, with levels $>40 \mathrm{IU} / \mathrm{ml}$ categorized as positive. Genotyping for rs6920220, rs13207033, and rs5029937 was performed using the Sequenom iPlex platform as described $^{2}$. Genotyping for DRB 1 alleles was performed using polymerase chain reaction-based methods. The following DRB1 alleles were classified as shared-epitope alleles: $* 0101, * 0102, * 0104, * 0401, * 0404, * 0405$, $* 0408, * 0409, * 0410, * 0413, * 0416, * 0419, * 0421$, and $* 1001$
Association between radiological damage and $6 q 23$ genotypes. Since this was a cross-sectional cohort, and modified Larsen scores (LS) were not normally distributed across the population (assessed by the Shapiro-Francia test for normality), comparison of median LS between genotype groups was performed using negative binomial regression, with disease duration, disease-modifying treatment, sex, and shared-epitope status included in the model. Subjects with missing data were excluded from analysis. A dominant model was chosen for analysis, due to the low frequency of the minor genotype for each SNP. Further analysis was performed after stratification for RF and anti-CCP antibody status. Association between radiographic severity and significantly associated SNP in combination was then assessed by comparing median LS according to carriage of statistically associated $(p<0.05)$ risk genotypes, referenced to the lowest risk combination (carriage of the protective allele at rs13207033, and absence of the risk alleles at rs6920220). All statistical analyses were carried out using Stata software (Release 9.2, Stata Corp., College Station, TX, USA).

\section{RESULTS}

Patients. Included in the study were 927 patients meeting 1987 ACR criteria for a diagnosis of RA. Characteristics of all participants are detailed in Table 1. Evidence of at least one radiographic erosion was found in $88.7 \%$ of patients.

Genotyping. Genotyping data for rs5029937, rs13207033, and rs692022 were available on 862 (93\%), 886 (95.5\%), and 916 (98.8\%) patients, respectively. All genotyping results conformed to Hardy-Weinberg equilibrium using the chi-square test for goodness of fit (Table 2). Genotype frequencies for each SNP were also compared according to anti-CCP antibody status using the chi-square test (for rs6920220 and rs13207033) and Fisher's exact test (for

Table 1. Patient characteristics. Values in parentheses are percentages.

\begin{tabular}{lc}
\hline Characteristic & RA Patients, $\mathrm{n}=927$ \\
\hline RF+ (available in $852 \mathrm{pts})$ & $580(68.1)$ \\
CCP+ (available in $909 \mathrm{pts})$ & $700(77.0)$ \\
Female & $674(72.7)$ \\
Mean age, yrs & 61.4 \\
Mean disease duration, yrs & 15.1 [range 3-47] \\
Erosive disease & $823(88.7)$ \\
Smoking & \\
Never & $387(41.8)$ \\
Previous & $352(38.1)$ \\
Current & $186(20.1)$ \\
Shared-epitope & \\
0 & $67(7.2)$ \\
1 copy & $324(35.7)$ \\
2 copies & $525(57.2)$ \\
Treatment & \\
No DMARD & $97(10.5)$ \\
MTX alone & $214(23.1)$ \\
Other DMARD* & $317(34.2)$ \\
MTX combination & $229(24.7)$ \\
Anti-TNF & $70(7.5)$ \\
Prednisolone & $495(53.3)$ \\
\hline
\end{tabular}

* Alone or in combination, including sulfasalazine, leflunomide, cyclosporine, gold, and azathioprine. ${ }^{\dagger}$ Shared-epitope status available in 916 patients. RF: rheumatoid factor; CCP: cyclic citrullinated peptide; DMARD: disease-modifying antirheumatic drug(s); MTX: methotrexate; TNF: tumor necrosis factor. 
Table 2. Genotype frequencies for $6 \mathrm{q} 23$ polymorphisms in patients with rheumatoid arthritis, and according to anti-CCP status.

\begin{tabular}{|c|c|c|c|c|c|}
\hline \multirow[t]{2}{*}{ SNP } & \multirow[t]{2}{*}{ Genotype } & \multicolumn{3}{|c|}{$\mathrm{N}$ (frequency) } & \multirow[b]{2}{*}{$\mathrm{p}^{*}$} \\
\hline & & $\begin{array}{c}\text { Whole Cohort, } \\
n=927\end{array}$ & $\begin{array}{l}\mathrm{CCP}+ \\
\mathrm{n}=700\end{array}$ & $\begin{array}{l}\mathrm{CCP}- \\
\mathrm{n}=209\end{array}$ & \\
\hline \multirow[t]{3}{*}{ rs6920220 } & $\mathrm{G} / \mathrm{G}$ & $507(0.55)$ & $379(0.55)$ & $117(0.56)$ & - \\
\hline & $\mathrm{G} / \mathrm{A}$ & $354(0.39)$ & $266(0.39)$ & $80(0.38)$ & - \\
\hline & $\mathrm{A} / \mathrm{A}$ & $55(0.06)$ & $43(0.06)$ & $12(0.06)$ & 0.95 \\
\hline \multirow[t]{3}{*}{ rs 13207033} & $\mathrm{G} / \mathrm{G}$ & $469(0.53)$ & $347(0.52)$ & $113(0.58)$ & - \\
\hline & $\mathrm{G} / \mathrm{A}$ & $359(0.41)$ & $281(0.42)$ & $71(0.36)$ & - \\
\hline & $\mathrm{A} / \mathrm{A}$ & $58(0.06)$ & $44(0.06)$ & $12(0.06)$ & 0.32 \\
\hline \multirow[t]{3}{*}{ rs5029937 } & $\mathrm{G} / \mathrm{G}$ & $776(0.90)$ & $587(0.89)$ & $174(0.94)$ & - \\
\hline & $\mathrm{G} / \mathrm{T}$ & $82(0.10)$ & $70(0.11)$ & $12(0.06)$ & - \\
\hline & $\mathrm{T} / \mathrm{T}$ & $3(0.00)$ & $3(0.00)$ & $0(0.00)$ & 0.20 \\
\hline
\end{tabular}

* Genotype frequencies for each SNP were compared according to anti-CCP antibody status using the chi-square test for rs6920220 and rs13207033, and Fisher's exact test for rs5029937. There was also no significant difference in genotype frequencies. SNP: single-nucleotide polymorphism; CCP: cyclic citrullinated peptide.

rs5029937) to ensure independence from autoantibody status. The markers were not in close linkage disequilibrium (rs5029937:rs13207033 $\mathrm{r}^{2}=0.01$, rs6920220:rs5029937 $\mathrm{r}^{2}$ $=0.01$, and $\mathrm{rs} 13207033$ :rs6920220 $\mathrm{r}^{2}=0.11$ ), and have been shown to reside on different haplotypes ${ }^{4}$.

Association between 6q23 genotype and radiographic damage. Association between genotype at each marker and median LS is shown in Table 3. In the cohort as a whole, there was no significant association between median LS and genotype at any of the markers. After stratification by autoantibody status, genotype at both rs6920220 and rs13207033 was associated with LS in patients positive for $\mathrm{RF}(\mathrm{p}=0.01$ and 0.05$)$ or anti-CCP antibodies ( $=0.02$ for both markers; Table 3), with higher LS in patients carrying the rare allele at rs6920220, and lower LS in patients carrying the rare allele at rs 13207033 . There was no association between genotype at any marker and LS in patients with autoantibody-negative disease.

Association between median LS and significantly associated SNP in combination was then assessed by comparing the lowest risk genotype combination (carriage of the protective allele at rs13207033, and absence of the risk allele at rs6920220) to other genotype combinations in autoanti- body-positive RA (Table 4). Comparison between the highest risk genotype (presence of risk allele at rs6920220 and absence of protective allele at rs 13207033) and the referent combination showed the strongest association: median LS 42 versus $29(\mathrm{p}=0.007)$ for $\mathrm{RF}+$ disease, and median LS 37 vs $28(\mathrm{p}=0.01)$ in anti-CCP+ patients. The associations were not strengthened by addition of genotype at rs 5029937 to the model (data not shown).

\section{DISCUSSION}

We have demonstrated association between genotype at rs6920220 and rs13207033, recently reported to be important susceptibility loci for RA, and radiographic severity in autoantibody-positive but not negative patients with RA. As with susceptibility, we have demonstrated that association between disease severity and these markers in combination is stronger than that for the markers individually.

One previous study examined association of genotypes at 6q23 and radiological severity in RA. As with our cohort, Scherer, et $a l^{12}$ found no effect of genotype at $6 \mathrm{q} 23$ polymorphisms and autoantibody-negative RA, but identified 2 markers, rs675520 and rs9376293, not in close linkage disequilibrium with the markers examined in this study, which

Table 3. Median Larsen score (n) according to genotype in $6 q 23$ risk markers, by autoantibody status. rs6920220 $0=\mathrm{G} / \mathrm{G}, 1=\mathrm{G} / \mathrm{A}$ and A/A; rs5029937 0 $=\mathrm{G} / \mathrm{G}, 1=\mathrm{G} / \mathrm{T}$ and $\mathrm{T} / \mathrm{T} ; \mathrm{rs} 132070330=\mathrm{G} / \mathrm{G}, 1=\mathrm{G} / \mathrm{A}$ and $\mathrm{A} / \mathrm{A}$

\begin{tabular}{|c|c|c|c|c|c|c|c|c|c|c|c|}
\hline Marker & Genotype & All & $\mathrm{p}^{*}$ & $\mathrm{RF}+$ & $\mathrm{p}$ & $\mathrm{RF}-$ & $\mathrm{p}$ & $\mathrm{CCP}+$ & $\mathrm{p}$ & $\mathrm{CCP}-$ & $\mathrm{p}$ \\
\hline \multirow[t]{2}{*}{ rs6920220 } & 0 & 25 (504) & - & 32 (307) & - & 15 (157) & - & 31 (377) & - & 14 (117) & - \\
\hline & 1 & $30(407)$ & 0.14 & $40(265)$ & 0.01 & $12(115)$ & 0.21 & $36(308)$ & 0.02 & $10(92)$ & 0.62 \\
\hline rs5029937 & 1 & $35(85)$ & 0.33 & $36.5(64)$ & 0.49 & $11(15)$ & 0.92 & $35(73)$ & 0.59 & $14.5(12)$ & 0.98 \\
\hline \multirow[t]{2}{*}{ rs 13207033} & 0 & 32 (467) & - & 39 (287) & - & 14 (139) & - & $37(345)$ & - & $14(113)$ & - \\
\hline & 1 & $25.5(414)$ & 0.12 & $32(269)$ & 0.05 & $15(121)$ & 0.84 & $29(324)$ & 0.02 & $10(83)$ & 0.84 \\
\hline
\end{tabular}

\footnotetext{
* For negative binomial regression, adjusted for disease duration, categorized disease-modifying treatment, sex, and shared-epitope status. RF: rheumatoid
} factor; CCP: cyclic citrullinated peptide. 
Table 4. Median Larsen scores, by combinations of the 2 most significant 6q23 markers.

\begin{tabular}{lcccccc}
\hline Genotype Combination & rs6920220 & rs13207033 & RF+ & p* & CCP+ & p \\
\hline 0 & 0 & 1 & $29(180)$ & - & $28(221)$ & - \\
1 & 0 & 0 & $38.5(116)$ & - & $38(142)$ & - \\
2 & 1 & 1 & $40(85)$ & - & $34(98)$ & - \\
3 & 1 & 0 & $42(169)$ & 0.007 & $37(200)$ & 0.01 \\
\hline
\end{tabular}

* For negative binomial regression with the genotype combination used as a categorical variable, adjusted for disease duration, categorized disease-modifying treatment, sex, and shared-epitope status. RF: rheumatoid factor; CCP: cyclic citrullinated peptide.

associated with radiographic damage. They found a trend for association at rs6920220, which did not meet statistical significance, but there was no association for rs 10499194, which is a perfect proxy for rs $13207033^{12}$. There are important differences between the cohorts studied - our patients were of much more advanced and variable disease duration and had received nonstandardized treatment regimens compared to the early-arthritis cohort, with serial radiographs included in the Scherer study, but the findings from both cohorts suggest an association with disease severity at $6 \mathrm{q} 23$ in autoantibody-positive RA, and the possibility that the true causal variant is yet to be identified. The influence of TNFAIP3 on disease severity is also supported by data from a murine study, which showed a very clear therapeutic effect of TNFAIP3 in mice with collagen-induced arthritis ${ }^{14}$.

The mechanism of association of rs6920220 and rs13207033 with RA is unclear at present. They map to a $60-\mathrm{kb}$ intergenic region of high linkage disequilibrium at 6q23.3, which does not encode any transcripts but does contain the PTPN11 pseudogene ${ }^{2}$. The block lies between the OLIG3 and TNFAIP3 genes. OLIG3 is a helix-loop-helix protein believed to be important in neuronal development and is not an obvious candidate gene. TNFAIP3, however, plays a central role in the regulation of immune signaling, acting as an inhibitor of nuclear factor- $\kappa \mathrm{B}$ activation, resulting in reduced production of proinflammatory cytokines such as tumor necrosis factor and $\mathrm{IL}-1^{16}$, and mice genetically deficient in TNFAIP3 have been shown to develop widespread tissue inflammation including a destructive arthritis ${ }^{17}$. A recent study has also shown a strong therapeutic effect of TNFAIP3 in mice with collagen-induced arthritis $^{14}$. TNFAIP3 is therefore a strong candidate to be the functional link for the disease associations identified in genetic studies at 6q23. Studies to date have not, however, identified an effect of genotype at the intergenic markers associated with autoimmune disease on TNFAIP3 expression. Intriguingly, recent work has shown that the subcellular expression of TNFAIP3 in synoviocytes may differ between RA and osteoarthritis, with reduced nuclear expression in patients with $\mathrm{RA}^{18}$, raising the possibility that reduced nuclear expression of TNFAIP3 may lead to dysregulated inflammatory activity in synoviocytes. The potential genetic influence on TNFAIP3 subcellular expression remains to be determined.
We wish to highlight several shortcomings to our study. First, the cohort was cross-sectional, containing patients of mixed disease duration and, as a consequence, there is a risk that differences in disease duration between the different genetic groups could lead to false-positive association between genotype and radiographic scores. All statistical tests were corrected for disease duration and other important confounders, but we appreciate that the cross-sectional design is a weakness. Second, while all patients were recruited from a single rheumatology department, treatments in this cross-sectional cohort have not been standardized, leading to the possibility of variance in radiological outcome as a consequence. Again, we are reassured by the lack of effect of this potential confounder in the multivariate model. Third, because this analysis is exploratory and the markers studied are not entirely independent of each other, $\mathrm{p}$ values are presented uncorrected, and, as a consequence, replication in large independent cohorts will be required to validate our findings. Finally, only white patients were included in this study, and the results cannot therefore be generalized to other ethnic groups.

We have demonstrated an association between 2 genetic markers at $6 \mathrm{q} 23$, previously identified to associate with susceptibility to RA, and radiographic disease severity in patients positive for RF or anti-CCP antibodies. As with susceptibility, markers at this locus in combination have a stronger association with disease severity than those in isolation. Our findings should be replicated in other large patient cohorts in order to validate them, and further studies are then needed to investigate the mechanisms underlying the associations we have demonstrated.

\section{REFERENCES}

1. Wellcome Trust Case Control Consortium. Genome-wide association study of 14,000 cases of seven common diseases and 3,000 shared controls. Nature 2007;447:661-78.

2. Thomson W, Barton A, Ke X, Eyre S, Hinks A, Bowes J, et al. Rheumatoid arthritis association at 6q23. Nat Genet 2007; 39:1431-3.

3. Plenge RM, Cotsapas C, Davies L, Price AL, de Bakker PI, Maller $\mathrm{J}$, et al. Two independent alleles at $6 \mathrm{q} 23$ associated with risk of rheumatoid arthritis. Nat Genet 2007;39:1477-82.

4. Orozco G, Hinks A, Eyre S, Ke X, Gibbons LJ, Bowes J, et al. Combined effects of three independent SNPs greatly increase the risk estimate for RA at 6q23. Hum Mol Genet 2009;18:2693-9.

5. Dieguez-Gonzalez R, Calaza M, Perez-Pampin E, Balsa A, Blanco Personal non-commercial use only. The Journal of Rheumatology Copyright $($ C 2012. All rights reserved. 
FJ, Canete JD, et al. Analysis of TNFAIP3, a feedback inhibitor of nuclear factor-kappa B and the neighbor intergenic 6q23 region in rheumatoid arthritis susceptibility. Arthritis Res Ther 2009;11:R42.

6. van der Helm-van Mil AH, Kern M, Gregersen PK, Huizinga TW. Variation in radiologic joint destruction in rheumatoid arthritis differs between monozygotic and dizygotic twins and pairs of unrelated patients. Arthritis Rheum 2006;54:2028-30.

7. Gregersen PK, Silver J, Winchester RJ. The shared epitope hypothesis. An approach to understanding the molecular genetics of susceptibility to rheumatoid arthritis. Arthritis Rheum 1987;30:1205-13.

8. Huizinga TW, Amos CI, van der Helm-van Mil AH, Chen W, van Gaalen FA, Jawaheer D, et al. Refining the complex rheumatoid arthritis phenotype based on specificity of the HLA-DRB1 shared epitope for antibodies to citrullinated proteins. Arthritis Rheum 2005;52:3433-8.

9. Marinou I, Healy J, Mewar D, Moore DJ, Dickson MC, Binks MH, et al. Association of interleukin- 6 and interleukin-10 genotypes with radiographic damage in rheumatoid arthritis is dependent on autoantibody status. Arthritis Rheum 2007;56:2549-56.

10. Cantagrel A, Navaux F, Loubet-Lescoulie P, Nourhashemi F, Enault $\mathrm{G}$, Abbal M, et al. Interleukin-1 beta, interleukin-1 receptor antagonist, interleukin-4, and interleukin-10 gene polymorphisms: Relationship to occurrence and severity of rheumatoid arthritis. Arthritis Rheum 1999;42:1093-100.

11. Buchs N, di Giovine FS, Silvestri T, Vannier E, Duff GW, Miossec P. IL-1B and IL-1Ra gene polymorphisms and disease severity in rheumatoid arthritis: Interaction with their plasma levels. Genes Immun 2001;2:222-8.
12. Scherer HU, van der Linden MP, Kurreeman FA, Stoeken-Rijsbergen G, le Cessie S, Huizinga TW, et al. Association of the $6 q 23$ region with the rate of joint destruction in rheumatoid arthritis. Ann Rheum Dis 2010;69:567-70.

13. Morgan AW, Robinson JI, Conaghan PG, Martin SG, Hensor EM, Morgan MD, et al. Evaluation of the rheumatoid arthritis susceptibility loci HLA-DRB1, PTPN22, OLIG3/TNFAIP3, STAT4 and TRAF1/C5 in an inception cohort. Arthritis Res Ther 2010;12:R57.

14. Hah YS, Lee YR, Jun JS, Lim HS, Kim HO, Jeong YG, et al. A20 suppresses inflammatory responses and bone destruction in human fibroblast-like synoviocytes and in mice with collagen-induced arthritis. Arthritis Rheum 2010;62:2313-21.

15. Mewar D, Coote A, Moore DJ, Marinou I, Keyworth J, Dickson $\mathrm{MC}$, et al. Independent associations of anti-cyclic citrullinated peptide antibodies and rheumatoid factor with radiographic severity of rheumatoid arthritis. Arthritis Res Ther 2006;8:R128.

16. Werner SL, Kearns JD, Zadorozhnaya V, Lynch C, O’Dea E, Boldin MP, et al. Encoding NF-kappa B temporal control in response to TNF: Distinct roles for the negative regulators I kappa B alpha and A20. Genes Dev 2008;22:2093-101.

17. Lee EG, Boone DL, Chai S, Libby SL, Chien M, Lodolce JP, et al. Failure to regulate TNF-induced NF-kappa B and cell death responses in A20-deficient mice. Science 2000;289:2350-4.

18. Elsby LM, Orozco G, Denton J, Worthington J, Ray DW, Donn RP. Functional evaluation of TNFAIP3 (A20) in rheumatoid arthritis. Clin Exp Rheumatol 2010;28:708-14. 


\section{Correction}

Rheumatoid Arthritis-associated Polymorphisms at 6q23 Are Associated with Radiological Damage in Autoantibody-positive RA

Maxwell J, Marinou I, Kuet K-P, Orozco G, Moore D, Barton A, Worthington J, Wilson G. Rheumatoid arthritis-associated polymorphisms at $6 \mathrm{q} 23$ are associated with radiological damage in autoantibody-positive RA. J Rheumatol 2012;9: 1781-5. Initials for 3 authors were not included in the article, and their names should have appeared as follows: JAMES R. MAXWELL, DAVID J. MOORE, and A. GERRY WILSON. We regret the error. doi:10.3899/jrheum.120144.C1 